PROCEEDINGS OF THE

AMERICAN MATHEMATICAL SOCIETY

Volume 137, Number 5, May 2009, Pages 1783-1791

S 0002-9939(08)09820-1

Article electronically published on December 23, 2008

\title{
ON INJECTIVITY OF QUASIREGULAR MAPPINGS
}

\author{
TADEUSZ IWANIEC, LEONID V. KOVALEV, AND JANI ONNINEN \\ (Communicated by Mario Bonk)
}

Abstract. We give sufficient conditions for a planar quasiregular mapping to be injective in terms of the range of the differential matrix.

\section{Introduction}

A holomorphic function $f: \Omega \rightarrow \mathbb{C}$ of one complex variable is a local homeomorphism if and only if $f^{\prime} \neq 0$ in $\Omega$. If, in addition, $\Omega$ is convex and $\operatorname{Re} f^{\prime} \geqslant 0$, then $f$ is either constant or injective in $\Omega$ [2]. In this paper we establish the analogues of these well-known facts for quasiregular mappings $f: \Omega \rightarrow \mathbb{C}$. By definition $f \in W_{\text {loc }}^{1,2}(\Omega)$ is quasiregular if there exists a constant $k<1$ such that $\left|f_{\bar{z}}\right| \leqslant k\left|f_{z}\right|$ a.e. in $\Omega$. Such a mapping can be called $K$-quasiregular with $K=(1+k) /(1-k)$, or $K$-quasiconformal if it is also injective.

Theorem 1.1. Let $\Omega \subset \mathbb{C}$ be a domain. If $f: \Omega \rightarrow \mathbb{C}$ is a nonconstant quasiregular mapping and $\operatorname{Re} f_{z} \geqslant 0$ almost everywhere, then $f$ is a local homeomorphism.

The proof is based on the celebrated theorem of Poincaré and Bendixson [5] and its extension by Brouwer 6, about local structure of integral curves of a continuous planar vector field near its critical point. Example 5.1 will show that the assumption $\operatorname{Re} f_{z} \geqslant 0$ cannot be replaced with $\left|\arg f_{z}\right| \leqslant \pi / 2+\epsilon$, for any $\epsilon>0$. To ensure that $f$ is injective in a convex domain $\Omega \subsetneq \mathbb{C}$ we must restrict the range of $f_{z}$ even further.

Theorem 1.2. Let $\Omega \subset \mathbb{C}$ be a convex domain. If $f: \Omega \rightarrow \mathbb{C}$ is a nonconstant quasiregular mapping and $\operatorname{Re} f_{z}=0$ almost everywhere, then $f$ is a homeomorphism.

This theorem admits the following reformulation (see section 31): if $\psi$ is a differentiable real-valued function on a convex domain $\Omega \subset \mathbb{R}^{2}$ and the gradient mapping $\nabla \psi: \Omega \rightarrow \mathbb{R}^{2}$ is quasiregular, then $\nabla \psi$ is either injective or constant. This is no longer true in dimensions $n \geqslant 3$, as is demonstrated by Example 5.3. Also, the assumption $\operatorname{Re} f_{z}=0$ in Theorem 1.2 cannot be replaced with $\left|\arg f_{z}\right|<\epsilon$, for any $\epsilon>0$, by Example 5.2. However, the situation is different when $\Omega=\mathbb{C}$. This can

Received by the editors July 21, 2008.

2000 Mathematics Subject Classification. Primary 30C62; Secondary 37C10, 30D20.

Key words and phrases. Quasiregular mapping, injectivity, differential inclusion.

The first author was supported by the NSF grant DMS-0800416.

The second author was supported by the NSF grant DMS-0700549.

The third author was supported by the NSF grant DMS-0701059.

(C)2008 American Mathematical Society 
be expected since by Picard's theorem an entire function whose derivative omits two values is linear and therefore is either constant or injective. For quasiregular mappings we have the following.

Theorem 1.3. If $f: \mathbb{C} \rightarrow \mathbb{C}$ is a non-constant quasiregular mapping and $\operatorname{Re} f_{z} \geqslant 0$ almost everywhere, then $f$ is a homeomorphism.

The sharpness of the assumption is demonstrated by Example [5.1] As a corollary of Theorems 1.1 and 1.3 we obtain a converse to the following theorem [4, Theorem 6.3.1]:

Theorem 1.4. If $f \in W_{\mathrm{loc}}^{1,2}(\mathbb{C})$ is a homeomorphic solution to the reduced Beltrami equation

$$
f_{\bar{z}}=\lambda(z) \operatorname{Re} f_{z}, \quad|\lambda(z)| \leqslant k<1,
$$

then $\operatorname{Re} f_{z}$ does not change sign.

Corollary 1.5. If $f \in W_{\text {loc }}^{1,2}(\mathbb{C})$ is a solution of (1.1) such that $\operatorname{Re} f_{z}$ does not change sign, then $f$ is a homeomorphism.

Let us emphasize that the notion of quasiregularity is invariant under an affine change of variables. Accordingly, the assumption $\operatorname{Re} f_{z} \geqslant 0$ in Theorems 1.1 and 1.3 can be replaced by somewhat geometrically pleasing conditions on the differential matrix $D f(z)$, which are also invariant under an affine change of variables. Let $\mathbb{R}^{2 \times 2}$ denote the 4-dimensional linear space of $2 \times 2$ matrices equipped with the inner product $\langle X, Y\rangle=\operatorname{Tr}\left(X Y^{*}\right)$, for $X, Y \in \mathbb{R}^{2 \times 2}$. Each nonzero matrix $N \in \mathbb{R}^{2 \times 2}$ gives rise to a 3 -dimensional subspace perpendicular to $N$,

$$
\mathbb{H}_{N}=\{X:\langle X, N\rangle=0\} \subset \mathbb{R}^{2 \times 2} .
$$

There are three types of such subspaces:

- $\mathbb{H}_{N}$ is said to be a positive subspace if $\operatorname{det} N>0$.

- $\mathbb{H}_{N}$ is said to be a negative subspace if $\operatorname{det} N<0$.

- $\mathbb{H}_{N}$ is said to be singular if $\operatorname{det} N=0$.

A 3-dimensional subspace $\mathbb{H}_{N}$ splits $\mathbb{R}^{2 \times 2}$ into two half-spaces. If $\operatorname{det} N>0$ we call them positive half-spaces of $\mathbb{R}^{2 \times 2}$. If $\operatorname{det} N<0$ we call them negative halfspaces. If $\operatorname{det} N=0$ the corresponding half-spaces are called singular half-spaces. Now, upon an affine change of variable, the assumption $\operatorname{Re} f_{z} \geqslant 0$ tells us that the essential range of $D f$ lies in a positive half-space. Precisely, this means that there is a constant matrix $N \in \mathbb{R}^{2 \times 2}$ of positive determinant such that

$$
\langle D f(z), N\rangle \geqslant 0 \quad \text { a.e. in } \Omega \text {. }
$$

As a matter of fact this amounts to saying that the homotopy between $f$ and the $\mathbb{R}$-linear map $L: \mathbb{R}^{2} \rightarrow \mathbb{R}^{2}$, with $D L=N$,

$$
f^{t}=(1-t) f+t L, \quad 0 \leqslant t \leqslant 1,
$$

keeps the distortion function of $f^{t}$ decreasing as $t$ increases from 0 to 1 . For example, if $L=$ id $: \mathbb{R}^{2} \rightarrow \mathbb{R}^{2}$, then condition (1.2) reads as $\operatorname{Re} f_{z} \geqslant 0$, so $f^{t}(z)=$ $(1-t) f(z)+t z$ and

$$
\left|\frac{f_{\bar{z}}^{t}}{f_{z}^{t}}\right|^{2}=\frac{\left|f_{\bar{z}}\right|^{2}}{\left(\operatorname{Re} f_{z}+\frac{t}{1-t}\right)^{2}+\left(\operatorname{Im} f_{z}\right)^{2}} \searrow 0 \text { as } t \nearrow 1 .
$$


The limit map $f^{1}(z)=z$ is a homeomorphism. Recall the Hurwitz-type theorems for quasiregular mappings; see [9, II 5.3] and [11, Lemma 3].

Theorem 1.6. If $f_{j}: \Omega \rightarrow \mathbb{C}$ is a sequence of [locally] $K$-quasiconformal mappings which converges uniformly on compact sets to $f: \Omega \rightarrow \mathbb{C}$, then $f$ is either constant or [locally] K-quasiconformal.

Now, heuristically, by virtue of Theorem 1.6 it should come as no surprise that Condition (1.2) yields local injectivity of $f$. However, our proof still requires the Poincaré-Bendixson analysis of the integral curves of the vector fields $f^{t}, 0<t \leqslant 1$. In view of these observations Theorem 1.1 is a statement on differential inclusions; let

$$
\mathbb{U}(K, N)=\left\{X \in \mathbb{R}^{2 \times 2}:|X|^{2} \leqslant K \operatorname{det} X,\langle X, N\rangle \geqslant 0\right\}
$$

for some $K \geqslant 1$ and $N \in \mathbb{R}^{2 \times 2}$ with positive determinant.

Every nonconstant solution to the differential inclusion

$$
D f(z) \in \mathbb{U}(K, N) \quad \text { a.e. in } \Omega, \quad f \in W_{\mathrm{loc}}^{1,2}(\Omega)
$$

is a local homeomorphism.

We refer the reader to 8 for a survey on differential inclusions.

\section{Proof of Theorem 1.1}

Let $f$ and $\Omega$ be as in the statement of Theorem 1.1. By virtue of Theorem 1.6, it suffices to prove that $f^{\lambda}(z):=f(z)+\lambda z$ is a local homeomorphism for $\lambda>0$. To simplify notation, we write $f$ instead of $f^{\lambda}$, keeping in mind that $\operatorname{Re} f_{z} \geqslant \lambda>0$ a.e. in $\Omega$. The local index of $f$ at $z_{0} \in \Omega$ is an integer defined by the rule

$$
n_{f}\left(z_{0}\right)=\frac{1}{2 \pi} \underset{0 \leqslant \theta \leqslant 2 \pi}{\Delta} \arg \left[f\left(z_{0}+r e^{i \theta}\right)-f\left(z_{0}\right)\right],
$$

where the increment of the argument of $f$ does not depend on the choice of radius $r$, provided $r$ is sufficiently small. It is a general topological fact that $f$ is locally injective if and only if $n_{f}\left(z_{0}\right)=1$ for every $z_{0} \in \Omega$. Since nonconstant quasiregular mappings are orientation-preserving [12, Theorem I.4.5], we have $n_{f}\left(z_{0}\right) \geqslant 1$. It remains to show that $n_{f}\left(z_{0}\right) \leqslant 1$ for every $z_{0} \in \Omega$. It involves no loss of generality in assuming that $z_{0}=0, f\left(z_{0}\right)=0, \Omega$ is the open unit disk, and $f(z) \neq 0$ for $0<|z| \leqslant 1$. Let $\Omega_{\circ}$ denote the punctured unit disk, $\Omega_{\circ}=\{z: 0<|z|<1\}$. We shall consider the integral curves of $f$ in $\Omega_{0}$, that is, solutions of the differential equation

$$
\frac{\mathrm{d} z}{\mathrm{~d} t}=f(z): 0<|z(t)|<1 \quad \text { for } a<t<b .
$$

By virtue of Peano's Existence Theorem, through every point $z_{0} \in \Omega_{\circ}$ there passes an integral curve, though uniqueness is not guaranteed. However, $z=z(t)$ can be extended (as a solution) over a maximal interval of existence, say $a_{-}<t<b_{+}$, $-\infty \leqslant a_{-}<b_{+} \leqslant \infty$. Moreover $z(t)$ tends to $\partial \Omega_{\circ}=\{0\} \cup \mathbb{S}^{1}$ as $t \rightarrow a_{-}$and $t \rightarrow b_{+}$. The extension of $z(t)$ need not be unique and the maximal interval of existence depends on the extension. Clearly $z=z(t)$ is of class $C^{1, \alpha}\left(a_{-}, b_{+}\right)$, $0<\alpha<1$. Since $f(0)=0$, it is possible in general that there is an injective solution $z=z(t) \in \Omega_{\circ}$ for $0 \leqslant t \leqslant \delta$ such that $\lim _{t \rightarrow \delta} z(t)=z(0)$. Then $\gamma=\{z(t): 0 \leqslant t<\delta\}$ is a rectifiable Jordan curve in $\Omega_{\circ}$. However, under our assumption, such curves are not present. Indeed, suppose such a $\gamma$ exists. To reach a contradiction, we let 
$\mathbb{U}$ denote the bounded component of $\mathbb{C} \backslash \gamma$; it is a simply connected region in $\Omega$. We integrate $f_{z}$ over $\Omega$ by using Stokes' theorem:

$$
\iint_{\mathbb{U}} f_{z} \mathrm{~d} x \mathrm{~d} y=\frac{1}{2 i} \iint_{\mathbb{U}} \mathrm{d}(f \mathrm{~d} \bar{z})=\frac{1}{2 i} \int_{\gamma} f \mathrm{~d} \bar{z}=\frac{1}{2 i} \int_{0}^{\delta}|f(z(t))|^{2} \mathrm{~d} t .
$$

This shows that

$$
\iint_{\mathbb{U}}\left(\operatorname{Re} f_{z}\right) \mathrm{d} x \mathrm{~d} y=0
$$

which is impossible since $\operatorname{Re} f_{z}>0$ almost everywhere.

Next we shall rule out the integral curves $\gamma=\left\{z(t) \mid a_{-}<t<b_{+}\right\}$such that $\lim _{t \rightarrow a_{-}} z(t)=\lim _{t \rightarrow b_{+}} z(t)=0$. Call such curves elliptic loops in $\Omega_{\circ}$. According to the celebrated Poincaré-Bendixson-Brouwer Theory [ [ such curves are present in every elliptic sector of $\Omega$. We shall not give a definition of an elliptic sector here as the need will not arise. The interested reader is referred to [5, 6, 7] for the definition and thorough discussion of sectors. The proof of nonexistence of elliptic loops is much the same as above. Adding the point 0 to $\gamma$ we obtain a Jordan curve, closure of $\gamma$ in $\Omega_{\circ}$. Let $\mathbb{U}$ denote the bounded component of $\mathbb{C} \backslash \bar{\gamma}$. To avoid delicate questions of rectifiability of $\gamma$ we remove from $\mathbb{U}$ a small disk $\mathbb{D}_{\epsilon}=\{z|| z \mid \leqslant \epsilon\} \subset \mathbb{U}$. Then we integrate as before:

$$
\iint_{\mathbb{U} \backslash \mathbb{D}_{\epsilon}} f_{z} \mathrm{~d} x \mathrm{~d} y=\frac{1}{2 i} \int_{\partial\left(\mathbb{U} \backslash \mathbb{D}_{\epsilon}\right)} f \mathrm{~d} \bar{z}=\frac{1}{2 i} \int_{\gamma_{1}} f \mathrm{~d} \bar{z}+\frac{1}{2 i} \int_{\gamma_{2}} f \mathrm{~d} \bar{z}
$$

where $\gamma_{1}=\partial \mathbb{U} \backslash \mathbb{D}_{\epsilon}$ and $\gamma_{2}=\mathbb{U} \cap \partial \mathbb{D}_{\epsilon}$. As before, the real part of the first integral term vanishes. The second term can be made as small as we wish. Indeed, we have

$$
\left|\frac{1}{2 i} \int_{\gamma_{2}} f \mathrm{~d} \bar{z}\right| \leqslant \frac{1}{2} \int_{|z|=\epsilon}|f||\mathrm{d} z|=\pi \epsilon\|f\|_{\infty} .
$$

Passing to the limit as $\epsilon \rightarrow 0$ we find that

$$
\int_{\mathbb{U}}\left(\operatorname{Re} f_{z}\right) \mathrm{d} x \mathrm{~d} y=0,
$$

which gives the desired contradiction.

Therefore, there are no elliptic sectors in $\Omega$. We now come to the fundamental theorem of Brouwer [6, Theorem 5], which asserts that the index of $f$ at the point 0 is given by

$$
n_{f}(0)=1+\frac{n_{e}-n_{h}}{2},
$$

where $n_{e}$ stands for the number of elliptic sectors and $n_{h} \geqslant 0$ stands for the number of hyperbolic sectors in $\Omega$. We just proved that $n_{e}=0$. Since $n_{h} \geqslant 0$ and $n_{f}(0) \geqslant 1$, this is only possible if $n_{h}=0$ and $n_{f}(0)=1$, as claimed.

\section{Proof of Theorem 1.2}

Let $f=u+i v$. In this notation the condition $\operatorname{Re} f_{z}=0$ reads as $u_{x}+v_{y}=0$. Therefore there exists a real-valued function $\psi$ such that

$$
\psi_{x}=-v \quad \text { and } \quad \psi_{y}=u
$$

or, using complex notation, $\nabla \psi=\psi_{x}+i \psi_{y}=i f$. For $(a, b) \in \Omega$ we define

$$
\psi^{a, b}(x, y)=\psi(x, y)-\left[\psi(a, b)+(x-a) \psi_{x}(a, b)+(y-b) \psi_{y}(a, b)\right] .
$$


Due to the local injectivity of $f,(a, b)$ is an isolated critical point of $\psi^{a, b}$. Since the topological index of $\nabla \psi^{a, b}(a, b)$ is equal to 1, by [1, Lemma 3.1] there is a neighborhood $U$ of $(a, b)$ such that either

(i) $\psi^{a, b}>0$ in $U \backslash(a, b)$ or

(ii) $\psi^{a, b}<0$ in $U \backslash(a, b)$.

We claim that only one of the above alternatives occurs for all $(a, b) \in \Omega$. Suppose to the contrary that (ii) occurs at $\left(a_{1}, b_{1}\right)$ and (iii) occurs at $\left(a_{2}, b_{2}\right)$. Consider the function $\phi(t)=\psi\left(a_{1}+t\left(a_{2}-a_{1}\right), b_{1}+t\left(b_{2}-b_{1}\right)\right)$ which is defined on some open interval containing $[0,1]$, because $\Omega$ is convex. Since any tangent line to the graph of $\phi$ stays (locally) on one side of the graph, the Mean Value Theorem implies that $\phi^{\prime}$ does not have any points of local extremum. Therefore, $\phi^{\prime}$ is monotone, and $\phi$ is either convex or concave. However, this contradicts our assumptions about $\left(a_{1}, b_{1}\right)$ and $\left(a_{2}, b_{2}\right)$.

Suppose for the sake of definiteness that only (ii) occurs in the entire domain $\Omega$. It follows that $\psi$ is strictly convex in $\Omega$. Being the gradient mapping of a strictly convex function, the map if $(z)$ is injective [13. Corollary 26.3.1], and so is $f$.

\section{Proof of Theorem 1.3}

Once we know that $f$ is locally quasiconformal, by Theorem 1.1, its global injectivity is a consequence of integral estimates near $\infty$. The following elementary, though interesting, fact yields Theorem 1.3 .

Proposition 4.1. If $f: \mathbb{C} \rightarrow \mathbb{C}$ is locally $K$-quasiconformal and $J_{f}(z)=\left|f_{z}\right|^{2}-$ $\left|f_{\bar{z}}\right|^{2} \geqslant \lambda^{2}$, almost everywhere for some $\lambda>0$, then $f$ is injective. Precisely we have

$$
\left|f\left(z_{1}\right)-f\left(z_{2}\right)\right| \geqslant \frac{\lambda}{\sqrt{K}}\left|z_{1}-z_{2}\right| .
$$

Proof. We may assume that $f(0)=0$ and $f(1)=1$, by rescaling if necessary. Stoilow factorization provides us with a normalized $K$-quasiconformal map $\chi$ : $\mathbb{C} \rightarrow \mathbb{C}, \chi(0)=0, \chi(1)=1$, such that $H(\omega)=f(\chi(\omega))$ is an entire function. We aim to show that $H(\omega) \equiv \omega$. Since $f$ is locally injective so is $H$. In particular, $H^{\prime}(\omega) \neq 0$. By the chain rule we have the following lower bound of the derivative:

$$
\left|H^{\prime}(\omega)\right|^{2}=J_{f}(z) J_{\chi}(\omega) \geqslant \lambda^{2} J_{\chi}(\omega) .
$$

Choose and fix a sufficiently small positive number $\epsilon$, for instance $0<\epsilon<\frac{1}{K-1}$ will suffice. Then we have

$$
\frac{1}{\left|H^{\prime}(\omega)\right|^{2 \epsilon}} \leqslant \frac{1}{\lambda^{2 \epsilon} J_{\chi}^{\epsilon}(\omega)}
$$

Consider the entire function

$$
F(\omega)=\left[H^{\prime}(\omega)\right]^{-\epsilon}=\sum_{m=0}^{\infty} a_{m} \omega^{m} .
$$

Integration over the disk $B=\{\omega|| \omega \mid \leqslant R\}$ yields

$$
\sum_{m=0}^{\infty} \frac{\left|a_{m}\right|^{2}}{m+1} R^{2 m}=f_{B}|F(\omega)|^{2} \mathrm{~d} \omega \leqslant \frac{1}{\lambda^{2 \epsilon}} \int_{B} \frac{\mathrm{d} \omega}{J_{\chi}^{\epsilon}(\omega)} .
$$

The integral average on the right-hand side exhibits a power growth with respect to $R$. Although the precise value of the power is immaterial for the forthcoming 
arguments, we demonstrate here the use of Astala's area distortion theorem, [3], to obtain a sharp power.

Lemma 4.2. If $\chi: \mathbb{C} \rightarrow \mathbb{C}$ is $K$-quasiconformal and $B \subset \mathbb{C}$ is a disk, then for every $0<\epsilon<\frac{1}{K-1}$ we have

$$
f_{B} \frac{\mathrm{d} \omega}{J_{\chi}^{\epsilon}(\omega)} \leqslant \frac{C_{K}}{1-(K-1) \epsilon}\left(\frac{|B|}{|\chi(B)|}\right)^{\epsilon} .
$$

In particular, if $\chi(0)=0$ and $\chi(1)=1$, then for $R \geqslant 1$,

$$
\frac{1}{\pi R^{2}} \int_{|\omega| \leqslant R} \frac{\mathrm{d} \omega}{J_{\chi}^{\epsilon}(\omega)} \leqslant C_{K}(\epsilon) R^{2 \epsilon(1-1 / K)} .
$$

Proof. See [4, Theorem 13.2.7].

We just arrived at the inequality

$$
\sum_{m=0}^{\infty} \frac{\left|a_{m}\right|^{2}}{m+1} R^{2 m} \leqslant \frac{C_{K}(\epsilon)}{\lambda^{2 \epsilon}} R^{2 \epsilon(1-1 / K)}
$$

where $2 \epsilon(1-1 / K)<2 / K<2$. Therefore, $a_{m}=0$ for $m \geqslant 1$. This means that $F(\omega)$ is constant, and so is $H^{\prime}(\omega)$. Hence $H(\omega)=\omega$ because of the normalization $H(0)=0$ and $H(1)=1$. In conclusion, $f(z)$ is the inverse of $\chi(\omega)$, and we have

$$
\begin{aligned}
& |D \chi(\omega)|^{2} \leqslant K J_{\chi}(\omega)=\frac{K}{J_{f}(z)} \leqslant \frac{K}{\lambda^{2}}, \\
& \left|\chi\left(\omega_{1}\right)-\chi\left(\omega_{2}\right)\right| \leqslant \frac{\sqrt{K}}{\lambda}\left|\omega_{1}-\omega_{2}\right|,
\end{aligned}
$$

which is equivalent to (4.1).

Returning to Theorem 1.3, we consider the quasiregular mappings

$$
f^{\lambda}(z)=f(z)+\lambda z, \quad \lambda \geqslant 0 .
$$

Clearly,

$$
J_{f^{\lambda}}(z)=J_{f}(z)+\lambda^{2}+2 \lambda \operatorname{Re} f_{z} \geqslant \lambda^{2} .
$$

Hence $f^{\lambda}$ is a $K$-quasiconformal mapping of $\mathbb{C}$ onto itself, for all $\lambda>0$. Passing to the limit as $\lambda \rightarrow 0$, by Theorem [1.6] we conclude that $f$ is injective in the entire plane.

\section{EXAMPLES}

Example 5.1. For every $\epsilon>0$ there is a nonconstant quasiregular map $f: \mathbb{C} \rightarrow \mathbb{C}$ whose $z$-derivative lies in the sector

$$
\operatorname{Re} f_{z} \geqslant-\epsilon\left|\operatorname{Im} f_{z}\right| \quad \text { a.e. in } \mathbb{C},
$$

and yet $f$ fails to be injective.

Proof. We need only consider $0<\epsilon \leqslant 2$. Let us introduce a parameter $\delta=$ $\frac{\epsilon}{2+\sqrt{4-\epsilon^{2}}} \leqslant \frac{\epsilon}{2} \leqslant 1$ so that $\epsilon=\frac{4 \delta}{1+\delta^{2}}$. First we define a quasiconformal homeomorphism of the upper half-plane $\mathbb{H}=\{z \in \mathbb{C}: \operatorname{Im} z>0\}$ onto the complex plane with 
a slit along the nonnegative $x$-axis:

$$
f(z)= \begin{cases}\frac{2 z^{2}}{|z| \sqrt{1+\delta^{2}}}, & \text { if } \operatorname{Re} z \geqslant-\delta \operatorname{Im} z \\ (i-\epsilon) z-i \bar{z}, & \text { if } \operatorname{Re} z \leqslant-\delta \operatorname{Im} z .\end{cases}
$$

A straightforward computation shows that

$$
(i-\epsilon) z-i \bar{z}=\frac{2 z^{2}}{|z| \sqrt{1+\delta^{2}}} \quad \text { on the ray } z=(i-\delta) t, t>0 .
$$

Thus $f$ is continuous on $\mathbb{H}$. Moreover, its complex derivatives outside this ray are

$$
\begin{aligned}
& f_{z}= \begin{cases}\frac{3 z}{|z| \sqrt{1+\delta^{2}}}, & \text { if } \operatorname{Re} z>-\delta \operatorname{Im} z, \\
i-\epsilon, & \text { if } \operatorname{Re} z<-\delta \operatorname{Im} z,\end{cases} \\
& f_{\bar{z}}= \begin{cases}\frac{-z^{3}}{|z|^{3} \sqrt{1+\delta^{2}}}, & \text { if } \operatorname{Re} z>-\delta \operatorname{Im} z, \\
-i, & \text { if } \operatorname{Re} z<-\delta \operatorname{Im} z .\end{cases}
\end{aligned}
$$

In any case we find that

$$
\left|f_{\bar{z}}\right| \leqslant \frac{1}{\sqrt{1+\epsilon^{2}}}\left|f_{z}\right|
$$

Regarding the condition (5.1), we have

$$
\operatorname{Re} f_{z} \geqslant \begin{cases}-\delta\left|\operatorname{Im} f_{z}\right|, & \text { if } \operatorname{Re} z>-\delta \operatorname{Im} z, \\ -\epsilon\left|\operatorname{Im} f_{z}\right|, & \text { if } \operatorname{Re} z<-\delta \operatorname{Im} z .\end{cases}
$$

In any case, $\operatorname{Re} f_{z} \geqslant-\epsilon\left|\operatorname{Im} f_{z}\right|$. Next we note that $f: \mathbb{H} \rightarrow \mathbb{C}$ extends continuously to the real line with values in the nonnegative real axis. Precisely, we have

$$
f(x+i 0)= \begin{cases}\frac{2 x}{\sqrt{1+\delta^{2}}}, & \text { for } x \geqslant 0, \\ -\epsilon x, & \text { for } x \leqslant 0 .\end{cases}
$$

By reflection about the $x$-axis, we extend $f$ to the lower half-plane, that is, by setting $f(z)=\overline{f(\bar{z})}$ if $\operatorname{Im} z \leqslant 0$. Inequality (5.3) holds almost everywhere in $\mathbb{C}$. But $f$ is no longer injective near any neighborhood of the origin, because

$$
f(z)=f(-z) \quad \text { if } \operatorname{Re} z \geqslant-\delta|\operatorname{Im} z| .
$$

It only remains to verify (5.1) in the lower half-plane. For $\operatorname{Im} z<0$ we have $f_{z}(z)=\overline{f_{z}(\bar{z})}$. Hence

$$
\operatorname{Re} f_{z}(z)=\operatorname{Re} f_{z}(\bar{z}) \geqslant-\epsilon\left|\operatorname{Im} f_{z}(\bar{z})\right|=-\epsilon\left|-\operatorname{Im} f_{z}(z)\right|=-\epsilon\left|\operatorname{Im} f_{z}(z)\right|
$$

as desired.

Example 5.2. Let $\Omega=\{z \in \mathbb{C}: \operatorname{Re} z>0\}$ be the right half-plane. For every $M \geqslant 1$ there is a nonconstant quasiregular map $f: \Omega \rightarrow \mathbb{C}$ such that

$$
\operatorname{Re} f_{z} \geqslant M\left|\operatorname{Im} f_{z}\right|
$$

a.e. in $\Omega$ and $f$ is not injective. 
Proof. For a given $M \geqslant 1$ we define

$$
f(z)= \begin{cases}\left(4 M^{2}+4 M i\right) z+\left(4 M^{2}+1\right) \bar{z}, & \text { if } 0<\operatorname{Re} z \leqslant 2 M \operatorname{Im} z, \\ \left(4 M^{2}-4 M i\right) z+\left(4 M^{2}+1\right) \bar{z}, & \text { if } 0<\operatorname{Re} z \leqslant-2 M \operatorname{Im} z, \\ \left(8 M^{2}-1\right) z, & \text { if } \operatorname{Re} z \geqslant 2 M|\operatorname{Im} z| .\end{cases}
$$

The reader may wish to verify that $f$ is quasiregular and satisfies inequality (5.4). Moreover, $f$ fails to be injective, because $f(1+4 M i)=1-8 M^{2}=f(1-4 M i)$.

Example 5.3. For any $n \geqslant 3$ there exists a function $\psi \in C^{1}\left(\mathbb{R}^{n}\right)$ such that $\nabla \psi: \mathbb{R}^{n} \rightarrow \mathbb{R}^{n}$ is a nonconstant quasiregular mapping that is not a local homeomorphism.

Proof. Define

$$
\psi\left(x_{1}, \ldots, x_{n}\right)=\frac{\left(x_{1}^{2}-x_{2}^{2}\right)^{2}-4 x_{1}^{2} x_{2}^{2}}{x_{1}^{2}+x_{2}^{2}}-\frac{x_{3}^{2}}{2}+\frac{1}{2} \sum_{k=4}^{n} x_{k}^{2} .
$$

Note that $\psi$ is homogeneous of degree 2 . Since $\psi$ is smooth away from the origin, the homogeneity implies that the entries of the Hessian matrix $D^{2} \psi$ belong to $L^{\infty}\left(\mathbb{R}^{n}\right)$. We claim that $\operatorname{det} D^{2} \psi \geqslant 16$ a.e. Thanks to the block-diagonal form of $D^{2} \psi$ and $\prod_{k=3}^{n} \frac{\partial^{2} \psi}{\partial x_{k}^{2}}=-1$ it suffices to show that $\operatorname{det} D^{2} u \leqslant-16$, where $u$ is the restriction of $\psi$ to the plane $\left\{x: x_{k}=0 \forall k \geqslant 3\right\}$. Writing $u$ in complex notation,

$$
u(z)=\frac{\operatorname{Re}\left(z^{4}\right)}{|z|^{2}}=\frac{1}{2}\left(\frac{z^{3}}{\bar{z}}+\frac{\bar{z}^{3}}{z}\right),
$$

we find that

$$
\nabla u=2 u_{\bar{z}}=\frac{3 \bar{z}^{2}}{z}-\frac{z^{3}}{\bar{z}^{2}}=\frac{\bar{z}^{3}}{|z|^{2}}\left(3-\frac{z^{4}}{\bar{z}^{4}}\right)
$$

and

$$
\operatorname{det} D^{2} u=4\left(\left|u_{\bar{z} z}\right|^{2}-\left|u_{\bar{z} \bar{z}}\right|^{2}\right)=-22-6 \operatorname{Re} \frac{z^{4}}{\bar{z}^{4}} \leqslant-16 \text {. }
$$

This proves that $\nabla \psi$ is quasiregular and, moreover, belongs to the class BLD (bounded length distortion) introduced by Martio and Väisälä 10. The last part of (5.5) shows that $\nabla u$ is homotopic to $z \mapsto \bar{z}^{3}$ in $\mathbb{C} \backslash\{0\}$. Since $\nabla u$ has index -3 at 0 , it follows that $\nabla u$ is not injective on any neighborhood of 0 . Consequently $\nabla \psi$ is not injective on any neighborhood of any point $x \in \mathbb{R}^{n}$ with $x_{1}=x_{2}=0$. In fact these points constitute the branch set of $\nabla \psi$, because $\nabla \psi$ is a local diffeomorphism elsewhere.

\section{ACKNOWLEDGEMENT}

We would like to thank the referee for a careful reading of the manuscript and several valuable comments which helped us to improve the presentation.

\section{REFERENCES}

1. G. Alessandrini and R. Magnanini, The index of isolated critical points and solutions of elliptic equations in the plane, Ann. Scuola Norm. Sup. Pisa Cl. Sci. (4) 19 (1992), no. 4, 567-589. MR:1205884 (94c:35063)

2. J. W. Alexander, Functions which map the interior of the unit circle upon simple regions, Ann. of Math. (2) 17 (1915), no. 1, 12-22. MR1503516

3. K. Astala, Area distortion of quasiconformal mappings, Acta Math. 173 (1994), no. 1, 37-60. MR 1294669 (95m:30028b) 
4. K. Astala, T. Iwaniec, and G. J. Martin, Elliptic partial differential equations and quasiconformal mappings in the plane, Princeton University Press, to appear.

5. I. Bendixson, Sur les courbes définies par des équations différentielles, Acta Math. 24 (1901), 1-88. MR.1554923

6. L.E.J. Brouwer, On continuous vector distributions on surfaces. II, Verhand. K. Ned. Akad. Wet. Afd. Nat. I. Reeks 12 (1910), 716-734. Available from http://www.digitallibrary.nl/ proceedings/

7. P. Hartman, Ordinary differential equations, John Wiley \& Sons, Inc., New York-LondonSydney, 1964. MR0171038 (30:1270)

8. B. Kirchheim, S. Müller, and V. Śverák, Studying nonlinear pde by geometry in matrix space, in "Geometric analysis and nonlinear partial differential equations", 347-395, Springer, Berlin, 2003. MR 2008346 (2006f:35087)

9. O. Lehto and K. I. Virtanen, Quasiconformal mappings in the plane, 2nd edition, SpringerVerlag, New York-Heidelberg, 1973. MR0344463 (49:9202)

10. O. Martio and J. Väisälä, Elliptic equations and maps of bounded length distortion, Math. Ann. 282 (1988), no. 3, 423-443. MR967022 (89m:35062)

11. R. Miniowitz, Normal families of quasimeromorphic mappings, Proc. Amer. Math. Soc. 84 (1982), no. 1, 35-43. MR633273 (83c:30026)

12. S. Rickman, Quasiregular mappings, Springer-Verlag, Berlin, 1993. MR1238941 (95g:30026)

13. R. T. Rockafellar, Convex analysis, Princeton University Press, Princeton, NJ, 1997. MR:1451876 (97m:49001)

Department of Mathematics, Syracuse University, Syracuse, New York 13244

E-mail address: tiwaniec@syr.edu

Department of Mathematics, Syracuse University, Syracuse, New York 13244

E-mail address: lvkovale@syr.edu

Department of Mathematics, Syracuse University, Syracuse, New York 13244

E-mail address: jkonnine@syr.edu 